\title{
(6) \\ Has the tobacco industry evaded the FDA's ban on 'Light' cigarette descriptors?
} OPEN ACCESS

\author{
Gregory N Connolly, Hillel R Alpert
}

Department of Social and Behavioral Sciences, Center for Global Tobacco Control, Harvard School of Public Health, Boston, Massachusetts, USA

\section{Correspondence to}

Professor Gregory N Connolly, Department of Social and Behavioral Sciences, Center for Global Tobacco Control, Harvard School of Public Health, 401 Park Drive, Landmark Building, Floor 4W, Room 403U, Boston, MA 02215, USA;

gconnoll@hsph.harvard.edu

Received 24 August 2012 Accepted 6 February 2013 Published Online First 13 March 2013

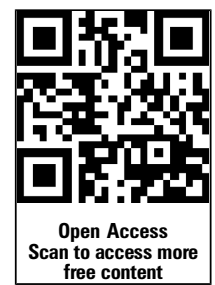

To cite: Connolly GN, Alpert HR. Tob Control 2014;23:140-145.

\section{ABSTRACT}

Background Under the Family Smoking Prevention and Tobacco Control Act (FSPTCA), the Food and Drug Administration (FDA) banned the use of "Lights" descriptors or similar terms on tobacco products that convey messages of reduced risk. Manufacturers eliminated terms explicitly stated and substituted colour name descriptors corresponding to the banned terms. This paper examines whether the tobacco industry complied with or circumvented the law and potential FDA regulatory actions.

Methods Philip Morris retailer manuals, manufacturers' annual reports filed with the Massachusetts Department of Public Health, a national public opinion survey, and market-wide cigarette sales data were examined.

Results Manufacturers substituted "Gold" for "Light" and "Silver" for "Ultra-light" in the names of Marlboro sub-brands, and "Blue", "Gold", and "Silver" for banned descriptors in sub-brand names. Percent filter ventilation levels, used to generate the smoke yield ranges associated with "Lights" categories, appear to have been reassigned to the new colour brand name descriptors. Following the ban, $92 \%$ of smokers reported they could easily identify their usual brands, and $68 \%$ correctly named the package colour associated with their usual brand, while sales for "Lights" cigarettes remained unchanged.

Conclusions Tobacco manufacturers appear to have evaded a critical element of the FSPTCA, the ban on misleading descriptors that convey reduced health risk messages. The FPSTCA provides regulatory mechanisms, including banning these products as adulterated (Section 902). Manufacturers could then apply for pre-market approval as new products and produce evidence for FDA evaluation and determination whether or not sales of these products are in the public health interest.

\section{INTRODUCTION}

The Food and Drug Administration (FDA) regulates industries accounting for nearly $25 \%$ of consumer spending in the USA for protection of the nation's public health and safety. ${ }^{1}$ On 23 June 2009 , the US President signed into law the Family Smoking Prevention and Tobacco Control Act (FSPTCA, the Act) extending the FDA authority to tobacco products, which take the lives of over 400000 Americans each year. ${ }^{2}$ The FSPTCA vests the FDA with the authority to set standards for tobacco products and the power as gatekeeper to control the entry of new products into the market, while products that were on the market prior to the Act's passage were allowed to remain. The significance of the FDA's new role is comparable with its authority over pharmaceuticals, which according to Professor Daniel Carpenter, member of the Institute of
Medicine advisory panel to the FDA, allows it 'to check and constrain a very large and politically dominant industrial sector'. ${ }^{3}$ Premarket approval is required for any tobacco product introduced after 15 February 2007 that is not deemed by the FDA to have substantial equivalence to a predicate product on the market as of that date. Importantly, Section 900 of the Act defines a brand as 'a variety of tobacco product distinguished by the tobacco used, tar content, nicotine content, flavoring used, size, filtration, packaging, logo, registered trademark, brand name, identifiable pattern of colors, or any combination of such attributes,', which allows the FDA to regulate the label and brand name as part of the tobacco product. ${ }^{2}$

This article examines the industry response to Section 911(b)(2)(ii) of the law, which came into effect on 22 June 2010, banning the use of explicit or implicit descriptors that convey messages of reduced risk including 'light', 'mild' and 'low', or similar descriptions in a tobacco product, label, labelling or advertising unless the manufacturer demonstrates to the FDA that the product significantly reduces harm and the risk of tobacco-related disease to individual tobacco users and benefits the health of the population as a whole. ${ }^{3}$ This action was based on findings, including a 2006 US federal court decision that the major US cigarette manufacturers were guilty under the Racketeer-Influenced and Corrupt Organization Act (RICO), citing 'longstanding and continuing fraudulent efforts to deceive the American public about 'light' and 'low tar' cigarettes', marketing them as less harmful when manufacturers knew they were not. ${ }^{4}$ Judge Gladys Kessler ruled, 'Consumers' false belief [of Lights] is so pervasive and longstanding and has been exploited and promoted by Defendants [tobacco manufacturers] for so long, that preventing and restraining Defendants' future fraud requires a ban on any future use of descriptors which convey a health message. ${ }^{4}$

Marketing of 'Light' cigarettes, a term used in this paper to include 'light' 'mild', 'medium', 'low', '1owest' and 'ultralight' cigarettes, surged after the first U.S. Surgeon General's 1964 report which found that cigarette smoking causes disease. ${ }^{5}$ During that time, tobacco manufacturers introduced filter ventilation, small holes placed before the machine attachment to lower tar and nicotine yields of cigarettes, using a standardised method developed by the Federal Trade Commission. Manufacturers used these measures to describe brands as light based on low yields and to convey messages of reduced risk despite the fact that the Federal Trade Commission method was neither intended nor able to predict actual human exposure 
to toxins. According to Philip Morris (PM), filter ventilation allows the mixing of air with smoke at controlled ratios and was used to classify cigarettes with 'Lights' and full-flavour categories: $0 \%-10 \%$ ventilation 'full flavour', 20\%-30\% ventilation 'light', 45\%-70\% ventilation 'ultra-light' and 60\%-75\% ventilation 'lowest'. 6

The 2001 National Cancer Institute (NCI) Monograph (Number 13) found that smokers compensate for the lower yield of Lights cigarettes by smoking more intensely, more often, or by fully or partially covering the ventilation holes to obtain a desired level of nicotine, thus negating any predictive value of Lights categories regarding disease risk. ${ }^{6}$ NCI also found that the manufacturers intentionally used Lights descriptors to market cigarettes as safer, and many smokers believed this message and switched in lieu of quitting. ${ }^{7}$ In the FSPTCA preamble, Congress stressed the public health significance of the Lights deception noting, "mistaken beliefs about the health consequences of smoking 'low tar' and 'light' cigarettes can reduce the motivation to quit smoking entirely and thereby lead to disease and death."2

Tobacco manufacturers responded to the ban by removing the terms explicitly listed in the law and substituting colour brand name descriptors that exactly corresponded to the banned Light descriptors. At the same time, they were appealing to the federal court, arguing that passage of the FSPTCA eliminated any reasonable likelihood that they would commit future RICO violations. ${ }^{8}$ However, following actions similar to the FSPTCA in other nations, manufacturers responded by using colour substitutes on packages as well as alternative terms. ${ }^{9-13}$ Subsequent studies found that consumers perceive colour descriptors on packaging as they do Lights descriptors, as less harmful to smoke than regular brands, ${ }^{14-18}$ and thus the colour descriptors are apt to perpetuate the Lights deception. The purpose of the present study is not to further examine consumer perceptions of risk associated with package colours and descriptors, but to address the urgent question for the FDA: Did Section 911 end the Lights deception as Congress intended, or has the provision been circumvented by the manufacturers' systematic colourname coding of Lights brands?

To address this question, this study examined three complementary lines of evidence: manufacturer intent reflected by brand name changes and marketing materials sent by PM to its retailers prior to the ban $^{19}$ and by manufacturers' annual reports required by Massachusetts Department of Public Health (MDPH) listing filter ventilation by full brand and sub-brand name; ${ }^{20}$ consumer response to the package changes based on a nationally representative survey of smokers conducted 1 year following the date of implementation; and market response based on national sales of Lights versus regular cigarettes before and after the ban. The combined evidence of this paper assesses tobacco industry compliance with the ban on Lights descriptors and regulatory actions available if the FSPTCA has been violated.

\section{METHODS}

Materials sent by PM to retailers in spring, 2010 were identified in the Legacy Tobacco Documents Library, ${ }^{19}$ and reviewed to determine company intent and manner of modifying Lights brand names in response to the ban. Manufacturers' annual reports of nicotine yield and related features of all products filed with MDPH were analysed for the years 2009-2011 focusing on the brand family with the largest market share by each of the major manufacturers: Marlboro by PM, Camel by Reynolds American, and Newport by Lorillard Tobacco, inclusive of 83 sub-brands for a total market share near $60 \% .^{21}$
A sub-brand in MDPH reports is specified as a unique combination of physical design and labelling characteristics. The numbers of sub-brands with a Lights descriptor and the numbers of sub-brands with one of the new typically used colour descriptors in the brand name, identified as described above, were tabulated for the years 2009 and 2011. Changes in these numbers were examined for the year just prior to and following the ban. The mean per cent filter ventilation among subbrands was computed and compared across Lights descriptor categories prior to the ban and across colour descriptor categories following the ban. A paired t test was used to test for a difference between per cent ventilation of PM sub-brands with Lights descriptors in 2009 prior to the ban and corresponding sub-brands in 2010 following the ban, using the PM retailer brochure which directly links new colour descriptors with the substituted Lights descriptors. ${ }^{19}$

A public opinion survey representative of the US adult population ages 18 years and older excluding Hawaii and Alaska was conducted via telephone from 18 May to 5 June 2011 by Social Science Research Solutions an independent research company, under contract with Harvard School of Public Health. The sample was obtained by bilingual random digit-dialling of landline telephones in households and of cell phone numbers, and with random selection of a single respondent within each household. This survey oversampled smokers and a minimum of 30 interviews was conducted in Spanish for representation of the Hispanic population. The response rate was 51\%. A total of 1021 completed the smoking component of the survey until reaching quotas of current smokers $(n=510)$ for the present study and non-smokers $(n=511)$ for other unrelated research. All data were weighted in a multi-phase design to adjust for the probability of selection and systematic non-response bias. The final weighting stage involved poststratification adjustment by raking so that the weighted sample reflects the US adult population along the lines of age-by-gender, race/ethnicity, education and census region. The survey was exempt from Institutional Review Board (IRB) approval. All respondents were asked about current and prior year smoking status in addition to demographics. Current smoking status was determined in the survey on the basis of responses to the questions, 'Have you smoked at least 100 cigarettes in your entire life?', and 'Do you now smoke cigarettes every day, some days or not at all?' Prior smoking status was determined on the basis of responses to the question, 'Six months ago, were you smoking cigarettes every day, some days, or not at all?' Smokers were first asked to name their usual cigarette brand based on the Lights descriptors banned a year before, and then asked three additional questions: 'In the past six months, how difficult has it been for you to identify your usual brand of cigarettes?'; 'Has it been very easy, somewhat easy, somewhat difficult, or very difficult?'; and 'What is the main color on the cigarette package of [your usual brand] that you smoked most often during the past 30 days?' Descriptive statistics incorporating the sampling weights were computed, and percentages and 95\% CIs are reported. The proportions of correctly identified package colours were compared across respondent usual brand descriptor categories using contingency table analysis and the Pearson $\chi^{2}$ statistic. All statistical analyses incorporated survey weights.

Cigarette sales and market share were derived from annual and quarterly ScanTrack scanner data licensed from ACNielsen ${ }^{22}$ for the years 2009 and 2010. Data for 2011 were not available at the time of the study. ScanTrack sampling is projected to represent approximately $95 \%$ of food and drug stores, $100 \%$ of convenience stores and $40 \%$ of mass merchandisers in the USA. 
PM sub-brands marketed with a colour in the brand name in 2009 prior to the ban were identified in the MDPH data. Sub-brands introduced with one of the new colour descriptors in the fourth quarter of 2010 following the ban were identified by the retailer brochure that directly links the new colour descriptors to the substituted Lights descriptors. The total 2009 and fourth quarter 2010 market shares of these colour named sub-brands were compared.

The total market shares of Marlboro, Camel and Newport sub-brands with Lights terms in the brand names in the first half of 2010 were compared with the total market shares of the corresponding brands linked by Universal Product Code (UPC) used for tracking trade items in stores in each of the last two quarters of 2010 following the ban.

\section{RESULTS}

\section{Brand name changes}

Examination of PM's retailer manual and analysis of the reports to MDPH confirmed that in response to the ban, the major cigarette manufacturers removed the terms explicitly stated in the law and substituted new colour terms for Lights brand name descriptors. The PM retailer manual states, 'current pack descriptors such as light, ultra-light and mild will be removed from all packaging' and new names were provided for each brand specified. 'Marlboro Light' sub-brands were renamed 'Marlboro Gold'. 'Marlboro Mild' sub-brands were renamed 'Marlboro Blue'. 'Marlboro Ultra-light' sub-brands were renamed 'Marlboro Silver'. ${ }^{19}$ The same colour substitutions occurred for Parliament, Virginia Slims, L\&M and Basic subbrands; while R.J. Reynolds (RJR) used 'Blue' for 'Camel Lights' sub-brands and 'Silver' for Ultra-light sub-brands; and Lorillard used 'Blue' and 'Gold' for 'Lights' sub-brands.

The numbers of Marlboro, Camel and Newport sub-brands with one of the colour descriptors Blue, Gold or Silver in the name increased over 10-fold from three in 2009 to 33 in 2010 as well as 2011, while the number of sub-brands with Lights descriptors dropped from 35 to zero in 2011 following the ban (figure 1). These Lights sub-brands, which were subsequently renamed with colour descriptors, represented $31.8 \%$ of the US cigarette market in $2009 .^{22}$

\section{Filter ventilation}

The mean per cent filter ventilation for Lights categories of the major brands in year 2009, prior to the ban, were graduated with levels that corresponded to the ventilation ranges of Lights categories described in PM internal industry documents, listing $20 \%-30 \%$ filter ventilation for light and $45 \%-70 \%$ filter

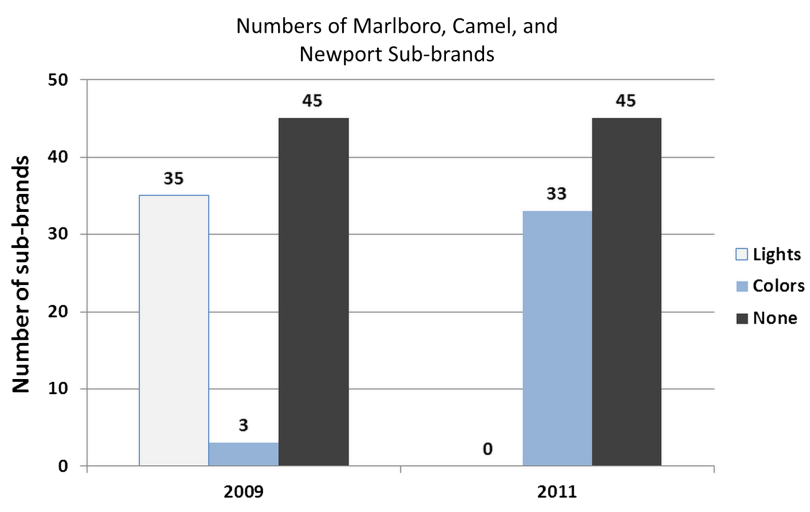

Figure 1 Numbers of sub-brands named by Lights and colour descriptors. ventilation for 'ultra-light' cigarettes (table 1). ${ }^{6}$ Prior to the ban, per cent filter ventilation levels did not correspond with colournamed descriptor categories. Filter ventilation was $13.0 \%$ for Marlboro Blue, 31.0\% for Camel Gold and 32.4\% for Camel Silver. In contrast, per cent filter ventilation did correspond with the Blue, Gold, and Silver colour brand name descriptors following the ban. Further, the same categories of per cent ventilation levels that were previously associated with Lights descriptors were associated with the postban colour brand name descriptor categories (table 1).

A comparison of the per cent filter ventilation levels among 30 discrete PM sub-brands with Lights descriptors in 2009 that were renamed with colour descriptors following the ban found no statistical significance (mean difference, 3.4 percentage points; $\mathrm{p}=0.701$ ) (table 2), indicating that per cent ventilation of these Lights sub-brands did not change when renamed with the new colour descriptors.

\section{Consumer response to package changes}

Current smoker respondents in the public opinion survey were mean age 43.2 years; $46.4 \%$ female; $66.4 \%$ White, $14.5 \%$ Black, Non-Hispanic and 10.7\% Hispanic; $51.1 \%$ married or living with a partner; $57.8 \%$ with high school or less education; and $56.3 \%$ had $\leq \$ 30000$ household income. Smokers identified their usual brand smoked as 51\% 'full flavour', 5\% 'medium', 31\% light, 7\% 'ultralight' and 4\% 'none of the above'.

One year after the ban, 88\%-91\% of smokers found it either 'somewhat easy' or 'very easy' to identify their usual brand of cigarettes by the banned descriptor names, Lights, Mediums or Ultra-lights. No statistically significant differences in the percentages were found across Lights and full-flavour categories, ranging from $88 \%$ to $92 \%$ (figure 2). In all, $68 \%$ of smokers correctly associated the substituted colour on their packages with the banned terms, ranging from 52.4\% among smokers of ultra-lights to $79.9 \%$ among smokers of full flavour/regular cigarettes $(\mathrm{p}<0.001)$ (figure 3$)$.

\section{Market response to package changes}

A total market share of $0.36 \%$ was found for the two PM subbrands that used colour brand name descriptors in 2009 and $30.3 \%$ market share for PM sub-brands that used one of the brand name colour descriptors in 2010 , for a nearly a 100 -fold increase. The total market share of the sub-brands with Light descriptors among Marlboro, Camel and Newport brands in the first two

Table 1 Mean per cent filter ventilation corresponding to 'Lights' and colour-named descriptor categories among major brands

\begin{tabular}{llll}
\hline & \multicolumn{2}{l}{ Mean per cent filter ventilation } \\
\cline { 2 - 4 } Category & Marlboro & Camel & Newport \\
\hline $\begin{array}{l}\text { Year: } 2009 \\
\text { Light }\end{array}$ & $29 \%$ & $32 \%$ & $23 \%$ \\
$\quad$ Ultra-light & $46 \%$ & $56 \%$ & - \\
Year: 2010 & & & \\
$\quad$ Blue & - & $29 \%$ & $28 \%$ \\
Gold & $29 \%$ & $38 \%$ & $34 \%$ \\
Silver & $50 \%$ & $40 \%$ & - \\
Year: 2011 & & & \\
$\quad$ Blue & - & $34 \%$ & $32 \%$ \\
Gold & $28 \%$ & $40 \%$ & $36 \%$ \\
Silver & $47 \%$ & $42 \%$ & - \\
\hline
\end{tabular}


Table 2 Comparison of ventilation in Philip Morris cigarette brands with colour names and the Lights brands replaced

\begin{tabular}{|c|c|c|c|c|}
\hline Sub-brand & Colour name substitute & Per cent ventilation 2009 & Per cent ventilation 2010 & $\Delta$ Per cent ventilation \\
\hline Basic lights 100s box & Gold & 23 & 16 & 7 \\
\hline Basic lights 100 s soft pack & Gold & 23 & 16 & 7 \\
\hline Basic lights box & Gold & 17 & 15 & 2 \\
\hline Basic lights soft pack & Gold & 16 & 15 & 1 \\
\hline Basic menthol lights 100 s box & Gold & 20 & 23 & 3 \\
\hline Basic menthol lights 100 s soft pack & Gold & 19 & 25 & 6 \\
\hline Basic menthol lights box & Gold & 16 & 17 & 1 \\
\hline Basic menthol lights soft pack & Gold & 12 & 14 & 2 \\
\hline Basic menthol ultra-lights 100 s box & Blue & 49 & 45 & 4 \\
\hline Basic menthol ultra-lights box & Blue & 42 & 39 & 3 \\
\hline Basic ultra-lights 100s box & Blue & 47 & 52 & 5 \\
\hline Basic ultra-lights 100 s soft pack & Blue & 50 & 48 & 2 \\
\hline Basic ultra-lights box & Blue & 41 & 42 & 1 \\
\hline Basic ultra-lights soft pack & Blue & 43 & 46 & 3 \\
\hline Marlboro lights 100 s box & Gold & 30 & 24 & 6 \\
\hline Marlboro lights 100 s soft pack & Gold & 30 & 24 & 6 \\
\hline Marlboro lights $25 \mathrm{~s}$ box & Gold & 18 & 25 & 7 \\
\hline Marlboro lights box & Gold & 25 & 23 & 2 \\
\hline Marlboro lights soft pack & Gold & 24 & 23 & 1 \\
\hline Marlboro medium 100s box & Red label & 20 & 23 & 3 \\
\hline Marlboro medium 100s soft pack & Red label & 25 & 21 & 4 \\
\hline Marlboro medium box & Red label & 18 & 17 & 1 \\
\hline Marlboro medium soft pack & Red label & 22 & 23 & 1 \\
\hline Marlboro ultra-lights 100 s box & Silver & 49 & 52 & 3 \\
\hline Marlboro ultra-lights box & Silver & 43 & 53 & 10 \\
\hline Merit lights 100 s soft pack & Gold & 33 & 32 & 1 \\
\hline Parliament lights 100 s soft pack & White & 30 & 34 & 4 \\
\hline Parliament lights box & White & 30 & 35 & 5 \\
\hline Parliament menthol lights box & White & 22 & 23 & 1 \\
\hline Virginia slims luxury lights 120 s box & Gold & 27 & 28 & 1 \\
\hline \multicolumn{4}{|c|}{ Mean absolute difference in per cent ventilation } & 3.4 \\
\hline
\end{tabular}

Sources: Philip Morris, USA ${ }^{7}$ and Annual Reports to Massachusetts Department of Public Health. ${ }^{18}$

quarters of 2010 (34\% and 33\%) was similar to that of sub-brands matched by UPCs in each of the second two quarters of 2010 (31\% and 30\%), showing no marked decline in annual sales.

\section{DISCUSSION}

Tobacco manufacturers appear to have circumvented the ban on Lights descriptors by intentionally substituting colour brand name descriptors, while maintaining the same gradient of ventilation used for Lights designations in the new colour-coding

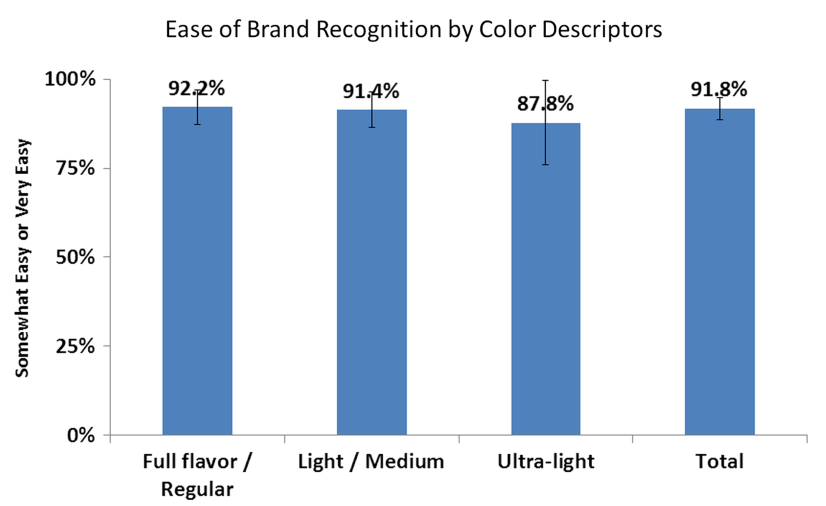

Figure 2 Percent of smokers reporting "somewhat easy" or "very easy" to identify their usual cigarettes by the banned descriptor names. scheme. The present findings appear to support the FDA's position in a letter to PM regarding the use of a Marlboro Lights package onsert prior to the ban. ${ }^{23}$ The onsert advised consumers regarding the pack changes and substitution of colour name descriptors as listed in the retailer manual. The FDA stated that the onsert suggests that Marlboro in the gold pack will have the same characteristics as Marlboro Lights, including any mistaken attributes associated with light cigarettes and may perpetuate the mistaken beliefs associated with these cigarettes. $^{24}$

The population survey found that 1 year following the ban smokers still perceived their usual brands as Lights or full flavour. While smokers of full flavour cigarettes were more often correct than smokers of Lights category brands in identifying their usual brand's pack colour, the majority of smokers of brands in all categories correctly identified their brands' pack colour. The NCI Monograph findings suggest that when smokers no longer perceive certain brands as Lights, fewer youth will initiate to low yield cigarettes and less switching from 'full flavour' to light cigarettes will occur. ${ }^{7}$ Consequently, a relative decrease in the sales of low yield cigarettes should be expected. However, national cigarette sales for Lights cigarettes did not change after the ban, suggesting the perpetuation of mistaken beliefs associated with these brands.

A review of industry practices following similar bans in other countries concluded that nations should ban Lights descriptors 


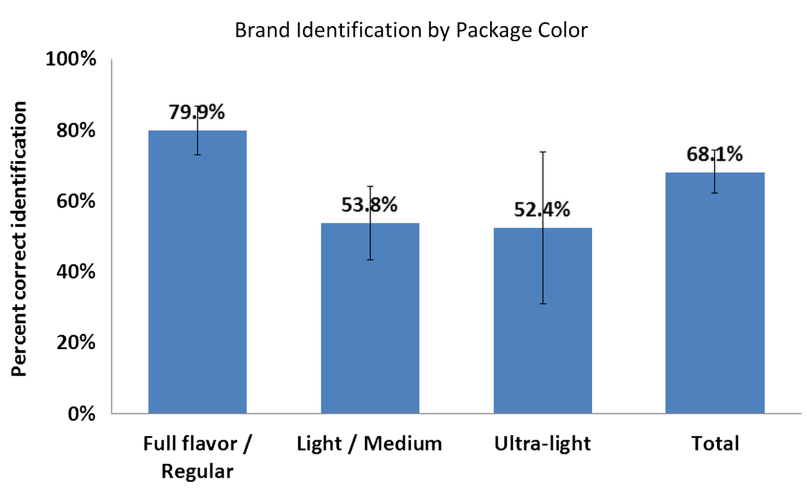

Figure 3 Percent of smokers correctly associating substituted colours with banned terms.

and 'misleading numbers, the use of colours, imagery, brand extensions, and other devices that contribute to deception'. ${ }^{24}$ The FDA appears to have the authority to do this based on the FSPTCA in order to end the deception of reduced risk associated with cigarette descriptor terms. The FDA's Draft Guidance in September 2011 states if 'the name of the cigarette brand was modified or changed, the cigarette is a new tobacco product and... the manufacturer must follow an appropriate regulatory pathway to market (i.e., a substantial equivalence report under 905(j), or a premarket [new] tobacco application under 910(b)). ${ }^{25}$ Substantial equivalence pertains to products marketed after 15 February 2007 and requires that the product has the same characteristics as a predicate product on the market as of that date or that the manufacturer demonstrate to the FDA that it does not raise different questions of public health. Similar to the language of substantial equivalence guidance for the FDA regulated medical devices, 'different questions' may be raised by changes in the product only and not necessarily by the product's negative effects. ${ }^{26}$ Different questions of public health are strongly suggested by: (a) manufacturers' substitution of new colour brand name descriptors for Lights, while maintaining the gradient of per cent ventilation levels associated with the brand name descriptors and (b) consumers' continued identification and smoking of these brands on the basis of the colour name descriptors.

Section 902 ('Adulterated Tobacco Products') allows the FDA to require removal of the modified brands and varieties from the market. ${ }^{2}$ Without a finding of substantial equivalence, the law requires manufacturers to submit premarket new tobacco product applications for the FDA approval prior to marketing modified brands. ${ }^{25}$ Manufacturers could remove all packaging changes not required by Section 911 and conduct testing to demonstrate to the FDA that the new products are appropriate for the protection of the public health and that the proposed packaging, labelling and design do not create false or misleading perceptions of safety. ${ }^{2}$

Other tobacco product characteristics in addition to labelling may contribute to false perceptions regarding risk, including concentrations of smoke constituents attributable to filter ventilation, and chemosensory effects. Colour-coded brand name descriptors associated with the ventilation of Lights appear to perpetuate the deception. Whether these descriptors maintain the false perception that such brands are 'safer' than others is not addressed in this study. Previous findings suggest that consumers do perceive brands with colours used as descriptors on packaging as being less harmful to smoke than regular brands. ${ }^{14-18}$ The present study is exploratory but does highlight the need for manufacturers to apply for premarket approval of these brands as new products in which they produce evidence that the FDA can evaluate and determine whether sales of these products are in the public health interest.

This study has limitations. Marketing materials of only one of the three major manufacturers were available for examination, the company with the largest market share. Annual reports of all manufacturers were available for examination, and coverage included all cigarette sub-brands whose brand families accounted for at least 3\% of market in respective years as required by the Massachusetts regulation. The public opinion survey did not ascertain whether respondents had their cigarette pack in hand when querying about its colour, which might have led to overestimation of their ability to identify pack colour. Smoking status of respondents was not verified with biomarkers, and their reported usual brand was not verified independently. ACNielsen ScanTrack data are comprehensive with respect to the food, drug and convenience store channels, but less so for mass merchandisers, although we are unaware of significant differences in market share in mass merchandisers that would account for the present findings. Finally, the postban market data in the last quarter of 2010 had not yet incorporated the new colour-named brand descriptors; nevertheless, comparisons made on the basis of UPC codes are believed to be reliable.

The findings of the present research strongly suggest that tobacco manufacturers have evaded one of the most important provisions of the FSPTCA for protecting the public health from the leading cause of preventable death and disease. The federal court has ordered tobacco companies to publicly admit that they have lied about the dangers of smoking in the past. ${ }^{27-29}$ Yet, far more important to the public health would be to ensure that the industry does not misuse Section 905(j) regarding substantial equivalence or other key provisions of the law or the FDA regulatory process to perpetuate public deception into the future.

\section{What this paper adds}

- By substituting colour brand name descriptors for banned Lights descriptors, tobacco manufacturers appear to have circumvented the FDA ban on misleading descriptors that convey messages of reduced health risk, a critical provision of the FSPTCA for protecting the public health from the leading cause of preventable death and disease.

- Ninety percent of smokers are still able to recognize their usual brand as "light" one year after the ban.

- The FPSTCA provides appropriate regulatory mechanisms to address the tobacco industry's perpetuation of the public deception concerning Lights cigarettes, including banning products that have evaded the law as adulterated (Section 902 ) and ensuring that the industry does not misuse Section 905(j) regarding substantial equivalence or other key provisions or FDA regulatory processes.

Contributors All authors contributed significantly to the conception, design and interpretation of data; drafting the article and revising it critically for important intellectual content; and final approval of the version to be published.

Funding This paper was funded by National Cancer Institute with a grant number of NCl 3R01 CA125224-03s1rev++ and NCI 2R01 CA087477-09A2.

Competing interests None.

Patient consent Patient consent was not applicable. 
Ethics approval Institutional review board approval was not required.

Provenance and peer review Not commissioned; externally peer reviewed.

Data sharing statement Data are not available for public sharing.

Open Access This is an Open Access article distributed in accordance with the Creative Commons Attribution Non Commercial (CC BY-NC 3.0) license, which permits others to distribute, remix, adapt, build upon this work non-commercially, and license their derivative works on different terms, provided the original work is properly cited and the use is non-commercial. See: http://creativecommons.org/ licenses/by-nc/3.0/

\section{REFERENCES}

1 FDA Science and Mission at Risk: Report of the Subcommittee on Science and Technology. FDA Science Board, Nov 2007. http://www.fda.gov/ohrms/dockets/ac/ 07/briefing/2007-329b_02_01_FDA\%20Report\%20on\%20Science\%20and\% 20Technology.pdf (accessed 24 aug 2012).

2 Family Smoking Prevention and Tobacco Control Act, Pub L. 111-31, 123 Stat 1776 (2009).

3 Carpenter D. Reputation and power: organizational image and pharmaceutical regulation at the FDA. New Jersey: Princeton University Press, 2010.

4 Final Opinion, United States v. Philip Morris USA, Inc. Civil Action No. 99-2496, 17 August 2006, page 1631. http://www.library.ucsf.edu/sites/all/files/ucsf_assets/ FinalOpinion_full_version.pdf (accessed 17 Jul 2012).

5 U.S. Public Health Service. Smoking and health: A report of the advisory committee to the Surgeon General of the Public Health Service. U.S. Department of Health, Education, and Welfare. Public Health Service. Centers for Disease Control. PHS Publication No. 1103. 1964.

6 Philip Morris. PM USA Product Development. Cigarette Manufacturing and Processing (PM Bates \#2063096854).

7 National Cancer Institute. Risks Associated with Smoking Cigarettes with Low Machine-Measured Yields of Tar and Nicotine. Smoking and Tobacco Control Monograph No. 13. Bethesda, MD: U.S. Department of Health and Human Services, National Institute of Health, National Cancer Institute, NIH Pub. No. 02-5074, October 2001.

8 United States v. Philip Morris USA Inc., et al Case number 11-5145, in the U.S. Court of Appeals for the District of Columbia Circuit. July 27, 2012.

9 Cavalcante T. (undated). Labeling and Packaging in Brazil. World Health Organization. http://www.who.int/tobacco/training/success_stories/en/ best_practices_brazil_labelling.pdf (accessed 22 Feb 2013).

10 Directive 2001/37/EC of the European Parliament and of the Council of 5 June 2001. Office Journal for the European Communities. 2001 July 11.

11 Scollo MM, Winstanley MH. eds. Tobacco in Australia: facts and issues. 3rd edn. Melbourne: Cancer Council Victoria, 2008. http://http://www.TobaccolnAustralia.org.au (accessed 22 Feb 2013).
12 Devlin E, Eadie D, Angus K. Low tar product category. Report prepared for NHS Health Scotland. Stirling, UK: The Centre for Tobacco Control Research, 2003.

13 King B, Borland R. What was "light" and "mild" is now "smooth" and "fine": new labeling of Australian cigarettes. Tob Control 2005;14:215.

14 Mutti S, Hammond D, Borland RM, et al. Beyond light and mild: cigarette brand descriptors and perceptions of risk in the International Tobacco Control (ITC) Four Country Survey. Addiction 2011;106:1166-75.

15 Borland R, Fong GT, Yong HH, et al. What happened to smokers' beliefs about light cigarettes when 'light/mild" brand descriptors were banned in the UK? Findings from the International Tobacco Control (ITC) Four Country Survey. Tob Control 2008;17:256-62.

16 Yong HH, Borland R, Cummings KM, et al. Impact of the removal of misleading terms on cigarette pack on smokers' beliefs about Light/Mild cigarettes: cross-country comparisons. Addiction 2011;106:2204-13.

17 Hammond D, Parkinson C. The impact of cigarette package design on perceptions of risk. J Public Health (Oxf) 2009:31:345-53.

18 Hammond D, Dockrell MM, Arnott D, et al. Cigarette pack design and perceptions of risk among UK adults and youth. Eur J Public health 2009;19:631-7.

19 Philip Morris, Retailer Brochure. Introducing New packaging on many Philip Morris USA (PM USA) brands. 2010. Bates Number 5003355067-5072.

20105 CMR 660.000. Cigarette and smokeless tobacco products: Reports of added constituents and nicotine ratings. Commonwealth of Massachusetts, Department of Public Health, Boston, MA.

21 Euromonitor International. Tobacco statistics. Retrieved from Euromonitor Passport GMID database. accessed 8 aug 2012).

22 ACNielsen. ScanTrack retail measurement, cigarette category, convenience store, food, drug, and mass merchandiser channels, Total US retail markets, 2009-2010. Schaumberg, IL, 2011.

23 Food and Drug Administration. Letter to Philip Morris USA, Inc., Marketing Marlboro Lights Cigarettes with an Onsert. Rockville MD, June 17, 2010.

24 Hoek J. An evaluation of regulatory responses governing the use of tobacco descriptors. Department of Marketing, Massey University, November, 2006. http:/l www.sfc.org.nz/resources/rrevaluationjanethoek.pdf (accessed 31 Jul 2011).

25 Draft Guidance for Industry and Food and Drug Administration Staff. Demonstrating the Substantial Equivalence of a New Tobacco Product: Responses to Frequently Asked Questions. September, 2011.

26 Draft Guidance for Industry and Food and Drug Administration Staff-The 510(k) Program: Evaluating Substantial Equivalence in Premarket Notifications [510(k)]. December 27, 2011.

27 Tobacco Companies Are Told to Correct Lies About Smoking, New York Times, November 28, 2012, Section B; Pg. 8.

28 Kendall B, Dooren JC. Tobacco Firms Told To Admit Deception. The Wall Street Journal, November 28, 2012, Section J; Pg. B3.

29 Frommer FJ. Judge orders tobacco companies to print statements they lied to public about smoking dangers. Associated Press Newswires, November 27, 2012. 